\title{
Position Control of a Maglev System Fed by a DC/DC Buck Power Electronic Converter
}

\author{
Victor Manuel Hernández-Guzmán $\mathbb{D}^{1},{ }^{1}$ Ramón Silva-Ortigoza $\mathbb{D},{ }^{2}$ \\ and Magdalena Marciano-Melchor $\mathbb{D}^{2}$ \\ ${ }^{1}$ Universidad Autónoma de Querétaro, Facultad de Ingeniería, Av. Hidalgo s/n, Centro Universitario, Cerro de Las Campanas, \\ C.P. 76010, Querétaro, Mexico \\ ${ }^{2}$ Instituto Politécnico Nacional, CIDETEC, Laboratorio de Mecatrónica \& Energía Renovable, C.P. 07700, \\ Ciudad de México, Mexico
}

Correspondence should be addressed to Victor Manuel Hernández-Guzmán; vmhg@uaq.mx

Received 4 April 2020; Accepted 11 May 2020; Published 31 May 2020

Guest Editor: Alberto Luviano Juárez

Copyright (C) 2020 Victor Manuel Hernández-Guzmán et al. This is an open access article distributed under the Creative Commons Attribution License, which permits unrestricted use, distribution, and reproduction in any medium, provided the original work is properly cited.

\begin{abstract}
In this paper, we solve the problem of position regulation in a magnetic levitation system that is fed by a DC/DC Buck power electronic converter as a power amplifier. We present a formal asymptotic stability proof. Although this result is local, the merit of our proposal relies on the fact that this is the first time that such a control problem is solved for a magnetic levitation system, a nonlinear electromechanical plant. In this respect, we stress that most works in the literature on control of electromechanical systems actuated by power electronic converters are devoted to control brushed DC motors which are well known to have a linear model. Furthermore, despite the plant that we control in the present paper is complex, our control law is simple. It is composed by four nested loops driven by one sliding mode controller, two proportional-integral controllers, and a nonlinear proportionalintegral-derivative position controller. Each one of these loops is devoted to control each one of the subsystems that compose the plant: electric current through the converter inductor, voltage at the converter capacitor, electric current through the electromagnet, and position of the ball. Thus, our proposal is consistent with the simple and intuitive idea of controlling each subsystem of the plant in order to render robust the control scheme. We stress that such a solution is complicated to derive using other control approaches such as differential flatness or backstepping. In this respect, our proposal relies on a novel passivitybased approach which, by exploiting the natural energy exchange between the mechanical and electrical dynamics, renders possible the design of a control scheme with the above cited features.
\end{abstract}

\section{Introduction}

One common technique that is used to supply power to electromechanical systems is pulse width modulation (PWM). However, the hard commutation that is intrinsic to PWM stresses the electromechanical system inducing abrupt changes in its dynamics which are observed as sudden variations in voltages and electric currents [1]. One manner to avoid this situation is the employment of DC/DC power electronic converters. Since these devices have embedded capacitors and inductors, they provide smooth voltages and electric currents, diminishing the effects of hard commutation in PWM-based power amplifiers.
The mathematical models of some DC/DC power electronic converter-DC motor systems were proposed for the first time in [2]. Since then, many works have been reported on control of several DC/DC power electronic converter topologies and DC motors [3-12]. Among the proposed control techniques are differential flatness, proportional-integral (PI) control, generalized PI control, passivity, adaptive control, PI fuzzy control, LQR (linearquadratic regulator) control, backstepping, and hierarchical control. The control problems that have been solved are unidirectional velocity regulation and tracking, velocity and torque control focusing on electrical transients, smooth velocity starters, and active disturbance rejection. In recent 
works [13-15], the introduction of an inverter between the DC/DC power electronic converter and the DC motor has rendered possible the bidirectional control of velocity.

The approach in [16], to control the DC/DC Buck power electronic converter-DC motor system, was inspired in part by [17-19]. Control scheme in [16] has the advantage of including a PI loop to control voltage at converter capacitor, a PI loop to control motor armature's current, and an external PI loop to regulate motor velocity. Hence, the main components of the successful strategies employed in industry to control electromechanical systems are included in the proposal of [16]. Moreover, another internal loop is devoted to control electric current through converter inductance. This loop is driven by a sliding mode control, a common strategy for control of power electronic converters in practice. The approach is proven in experiments to be robust with respect to parametric uncertainties and external disturbances.

On the contrary, magnetic levitation systems are commonly used as benchmark problems to test novel control approaches. Among the proposed control techniques, the passivity-based approaches presented in [20-22] have been welcomed in the control community. In particular, the solution presented in [20] is interesting because it possesses a classical proportional-integral-derivative (PID) controller to cope with the mechanical part of the system. However, since the design is performed in terms of magnetic flux, instead of electric current, efforts are oriented to avoid the implementation of any internal loop to cope with the electrical dynamics. This is because of the complications arising from magnetic flux measurements. In this respect, we stress that experimental results have been reported in the literature showing that such internal loop is necessary to improve performance in practice, see [23], for instance.

The novel control technique known as immersion and invariance (I\&I) has been employed in $[24,25]$ to control magnetic levitation systems. Novelty in those applications is that a (small) parasitic capacitance is considered to be present at terminals of the electromagnet. The main target to use I\&I in such a control problem is to extend the application of any control law, say $w$, that has been designed when such a parasitic capacitor is not present. However, since this requires to feedback the time derivative of $w$, the online computation of an important number of additional complex terms are required.

In the present paper, we extend the work in [16] to control the ball position in a magnetic levitation system which is fed by a DC/DC Buck power electronic converter. This implies that additional inductance and capacitance with considerable values are included in the electrical circuitry of the magnetic levitation system. Since a magnetic levitation system only requires unipolar voltage, such a power converter topology is adequate and any inverter is not required. We stress that a magnetic levitation system is a complex and nonlinear system. Hence, controlling for the first time and from a theoretical point of view, a plant with these features when it is fed by a DC/ DC Buck power electronic converter represents one important contribution of the present paper.
Despite the complex and nonlinear nature of the magnetic levitation system, our proposal is simple. It is composed by a PI loop to control voltage at the converter capacitor, a PI loop to control the electromagnet electric current, and an external PID loop to regulate the ball position. As in [16], an additional sliding modes' internal loop is employed to control electric current through the converter inductance. Formalizing this intuitively simple idea to control a complex plant is another important contribution of the present paper. The key for this is a novel passivity-based approach exploiting energy ideas, i.e., we take advantage from the natural energy exchange among the several subsystems to design the control law. This represents another contribution of the present paper.

This paper is organized as follows. In Section 2, we introduce the plant to be controlled and present its dynamical model. The passivity properties of the plant are described in Section 3 where we also give some insight on the rationale behind our approach. Our main result is presented in Section 4. In Section 5, we present a simulation study and, finally, some concluding remarks are given in Section 6.

\section{Mathematical Model}

The DC/DC Buck power electronic converter-Magnetic levitation system is depicted in Figure 1(a). The DC/DC Buck power converter is composed by a transistor $Q$, a diode $D$, an inductor $L_{c}$, a capacitor $C$, and a resistance $R_{c}$. Symbols $i_{c}$ and $v$ represent electric current through inductance $L_{c}$ and voltage at capacitor terminals $C$, respectively, whereas $E$ stands for voltage of the DC power supply. The system input is $u$ which only takes the discrete values $\{0,+1\}$ representing the off and on states of transistor $Q$, see Figure 1(b).

The magnetic levitation system consists of an electromagnet, with inductance $L(y)$ and internal resistance $R$, and a ball with mass $m$, made in a ferromagnetic material, which receives an upwards magnetic force $F$ from the electromagnet. This force must cancel the downwards ball weight $m g$ in order to levitate the ball in space. Electromagnet is basically a ferromagnetic core with a conductor wire wound around it. The electric voltage $v$ is applied at the electromagnet terminals which force an electric current $i$ to flow through the electromagnet winding and this current produces the attractive magnetic force $F=(1 / 2)(\mathrm{d} L(y) / \mathrm{d} y) i^{2}$ on the ball. Symbol $\lambda$ represents the magnetic flux produced by electric current $i$ within the electromagnet core. Ball position, measured from the bottom of the electromagnet to the top of ball is represented by $y \geq 0$. We remark that inductance of electromagnet, $L(y)>0$ for all $y \geq 0$, depends on the ball position $y$ in the form shown in Figure 2. In order to understand this, recall that the magnetic flux is given as $\lambda=L(y) i$. Suppose that $i$ remains constant and the ball approaches to electromagnet, i.e., $y$ decreases. This reduces both the air gap and the reluctance. Hence, $\lambda$ increases. Since $\lambda=L(y) i$ and $i$ remains constant, this means that $L(y)$ must increase. Thus, $L(y)$ increases as $y$ decreases. When $y \longrightarrow \infty$, it is obtained as the case when the ball is not 
present, and in such a case, $L(y)$ reaches a minimum positive value. This also means that $\mathrm{d} L(y) / \mathrm{d} y<0$.

We refer the reader to [23] for a detailed description of a magnetic levitation system as well as for precise instructions to construct one of them for experimental purposes. Furthermore, the complete procedure to obtain its dynamical model is presented and some experiments are provided to identify its parameters. Also, some controllers are designed and tested experimentally.

Using Kirchhoff's Laws (see Figure 1(b)), Faraday's Law, and Newton's Second Law, we find that the mathematical model of the DC/DC Buck power electronic convertermagnetic levitation system is given as follows $[16,23,26,27]$ :

$$
\begin{gathered}
L_{c} \frac{\mathrm{d} i_{c}}{\mathrm{~d} t}=-v+E u, \\
C \frac{\mathrm{d} v}{\mathrm{~d} t}=i_{c}-i-\frac{v}{R_{c}}, \\
L(y) \frac{\mathrm{d} i}{\mathrm{~d} t}=-\frac{\mathrm{d} L(y)}{\mathrm{d} y} i \frac{\mathrm{d} y}{\mathrm{~d} t}-R i+v, \\
m \ddot{y}=\frac{1}{2} \frac{\mathrm{d} L(y)}{\mathrm{d} y} i^{2}+m g, \\
\frac{\mathrm{d} L(y)}{\mathrm{d} y}<0, \quad \forall y \geq 0 .
\end{gathered}
$$

Important for our purposes is the following class of saturation functions.

Definition 1. Given positive constants $L^{*}$ and $M$, with $L^{*}<M$, a function $\sigma: \mathscr{R} \longrightarrow \mathscr{R}: \varsigma \mapsto \sigma(\varsigma)$ is said to be a strictly increasing linear saturation for $\left(L^{*}, M\right)$ if it is locally Lipschitz, strictly increasing, and satisfies [28]

$$
\begin{aligned}
\sigma(\varsigma) & =\varsigma, \quad \text { when }|\varsigma| \leq L^{*}, \\
|\sigma(\varsigma)| & <M, \quad \forall \varsigma \in \mathscr{R} .
\end{aligned}
$$

\section{The Rationale behind Our Proposal}

Consider the following slightly modified version of the mathematical model in (1)-(5):

$$
\begin{aligned}
C \frac{d v}{d t} & =i_{c}-i-\frac{v}{R_{c}}, \\
L(y) \frac{\mathrm{d} i}{\mathrm{~d} t} & =-\frac{\mathrm{d} L(y)}{\mathrm{d} y} i \dot{y}-R i+v, \\
m \ddot{y} & =\frac{1}{2} \frac{\mathrm{d} L(y)}{\mathrm{d} y} i^{2}-G(y),
\end{aligned}
$$

where $G(y)=\mathrm{d} P(y) / \mathrm{d} y$ with $P(y)$ is a positive semidefinite scalar function. The total energy stored in the system is given as follows:

$$
V_{e}(v, y, \dot{y}, i)=\frac{1}{2} C v^{2}+\frac{1}{2} L(y) i^{2}+\frac{1}{2} m \dot{y}^{2}+P(y),
$$

where the first term stands for electric energy stored in the capacitor of the Buck power converter, whereas the last three terms stand for the magnetic, kinetic, and potential energies stored in the electrical and the mechanical subsystems, respectively, of the magnetic levitation system. The time derivative of $V_{e}$ along the trajectories of system in (7) is given as follows:

$$
\begin{aligned}
\dot{V}_{e}= & v C \frac{\mathrm{d} v}{\mathrm{~d} t}+\frac{1}{2} \frac{\mathrm{d} L(y)}{\mathrm{d} y} \dot{y} i^{2}+i L(y) \frac{\mathrm{d} i}{\mathrm{~d} t}+\dot{y} m \ddot{y}+\frac{\mathrm{d} P(y)}{\mathrm{d} y} \dot{y} \\
= & v\left[i_{c}-i-\frac{v}{R_{c}}\right]+\frac{1}{2} \frac{\mathrm{d} L(y)}{\mathrm{d} y} \dot{y} i^{2}+i\left[-\frac{\mathrm{d} L(y)}{\mathrm{d} y} i \dot{y}-R i+v\right] \\
& +\dot{y}\left[\frac{1}{2} \frac{\mathrm{d} L(y)}{\mathrm{d} y} i^{2}-G(y)\right]+G(y) \dot{y} \\
= & -\frac{v^{2}}{R_{c}}-R i^{2}+i_{c} v,
\end{aligned}
$$

Notice that the cancellation of terms

$$
\begin{aligned}
\frac{1}{2} \frac{\mathrm{d} L(y)}{\mathrm{d} y} \dot{y} i^{2}-\frac{\mathrm{d} L(y)}{\mathrm{d} y} i^{2} \dot{y}+\frac{1}{2} \frac{\mathrm{d} L(y)}{\mathrm{d} y} \dot{y} i^{2} & =0, \\
i v-v i & =0,
\end{aligned}
$$

represent (1) natural energy exchange between the electrical and the mechanical subsystems of the magnetic levitation system and (2) natural energy exchange between the capacitor and the electrical subsystem of the magnetic levitation system. Another cancellation of the terms involves $\pm G(y) \dot{y}$ which represents the exchange between kinetic and potential energies in the magnetic levitation system. Hence, if we define the input $i_{c}$ and the output $v$, then

$$
\dot{V}_{e} \leq-\frac{v^{2}}{R_{c}}+v i_{c} .
$$

The expression in (11) proves that the model in (7) is output strictly passive [29], Definition 6.3.

In the present paper, we exploit these properties by proceeding as follows. First, we design $u$ as a sliding mode controller to force $i_{c}$ to reach a desired function $i_{c}^{d}$. Then, $i_{c}=i_{c}^{d}$ is employed as the control input for the sliding surface systems (2)-(4). In order to perform this step, we first obtain the error equation for this system by adding and subtracting some convenient terms (notice that these terms are not introduced using any control law), i.e.,

$$
\begin{aligned}
C \frac{\mathrm{d} \varepsilon}{\mathrm{d} t}= & i_{c}^{d}-\widetilde{I}-i^{d}-\frac{\varepsilon}{R_{c}}-\frac{v^{d}}{R_{c}}-C \frac{\mathrm{d} v^{d}}{\mathrm{~d} t}, \\
L(y) \frac{\mathrm{d} \widetilde{I}}{\mathrm{~d} t}= & -\frac{\mathrm{d} L(y)}{\mathrm{d} y} \widetilde{I} \frac{\mathrm{d} y}{\mathrm{~d} t}-\frac{\mathrm{d} L(y)_{i d} d \frac{\mathrm{d} y}{\mathrm{~d} y}-R \widetilde{I}-R i^{d}}{\mathrm{~d} t} \\
& +\varepsilon+v^{d}-L(y) \frac{\mathrm{d} i^{d}}{\mathrm{~d} t}, \\
m \ddot{y}= & \frac{1}{2} \frac{\mathrm{d} L(y)}{\mathrm{d} y} \widetilde{I}^{2}+\frac{\mathrm{d} L(y)^{d}}{\mathrm{~d} y} i^{d} \widetilde{I}+\frac{1}{2} \frac{\mathrm{d} L(y)^{d}}{\mathrm{~d} y} i^{d 2}+m g,
\end{aligned}
$$




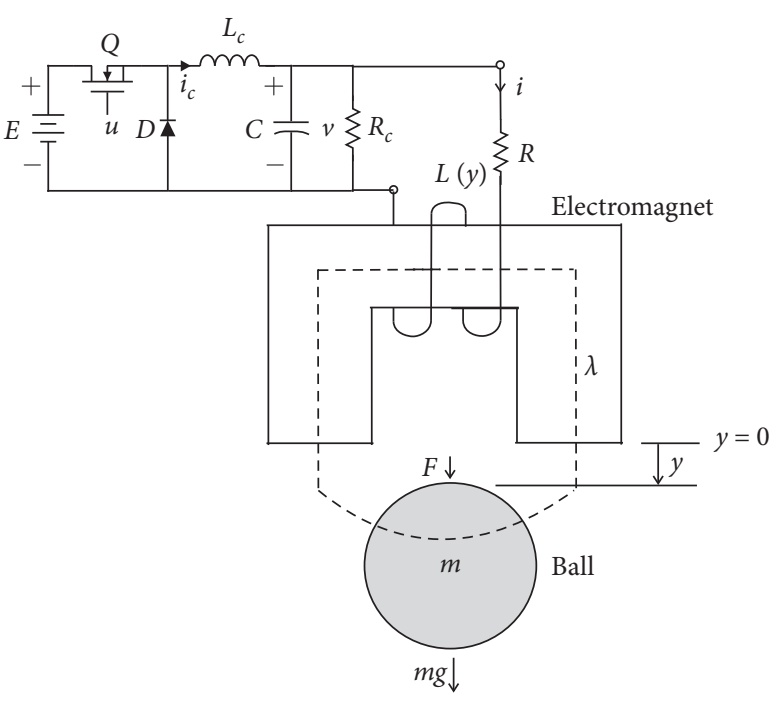

(a)

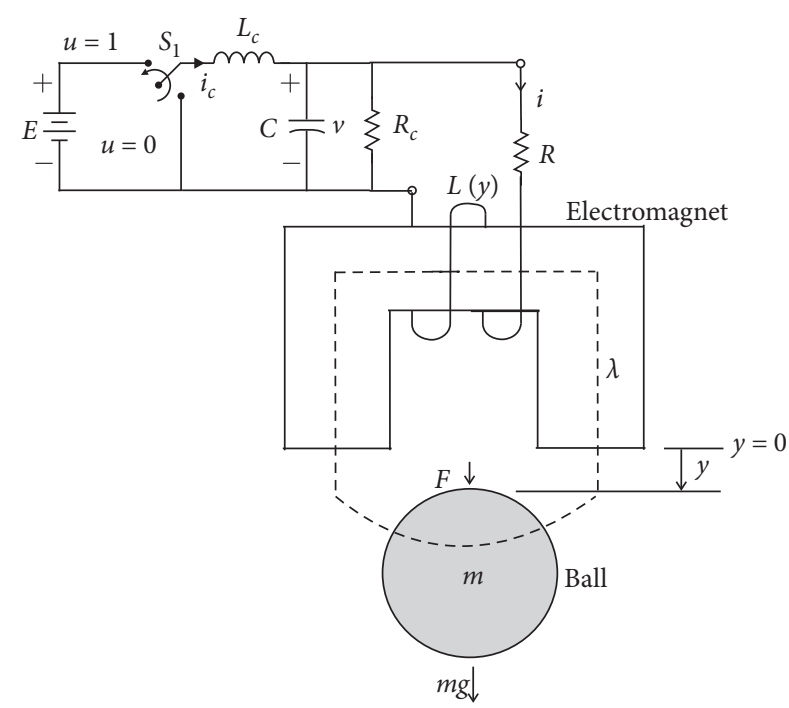

(b)

FIGURE 1: Electromechanical diagram of the DC/DC Buck power electronic converter-magnetic levitation system. (a) Implementation of the DC/DC Buck power electronic converter-magnetic levitation system using one diode and one transistor. (b) Ideal representation of the DC/ DC Buck power electronic converter-magnetic levitation system using a switch $S_{1}$.

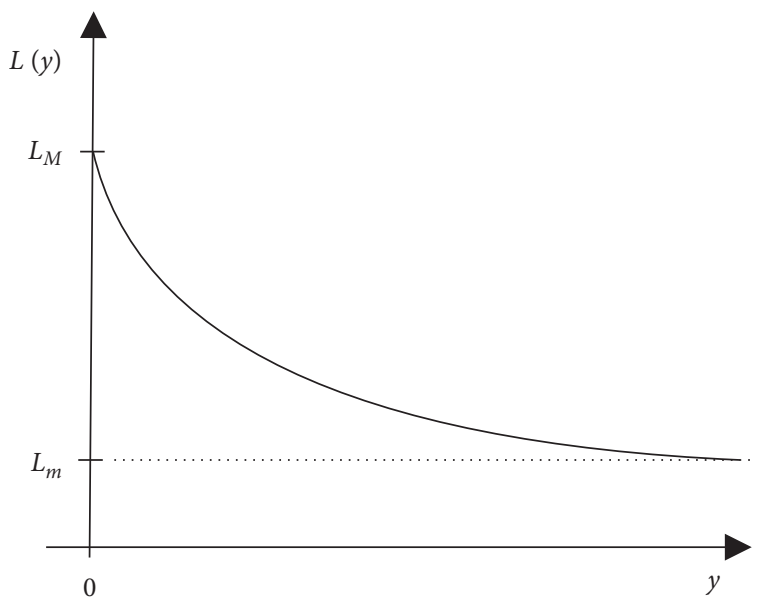

Figure 2: Inductance $L$ as a function of the ball position $y$.

where $\varepsilon=v-v^{d}$ and $\widetilde{I}=i-i^{d}$. The equivalence of these expressions and those in (2)-(4) can be verified by reducing the redundant terms in the three expressions in (12). Defining $V_{E 1}(\varepsilon, \widetilde{I}, \dot{y})=(1 / 2) C \varepsilon^{2}+(1 / 2) L(y) \widetilde{I}^{2}+(1 / 2) m \dot{y}^{2}$ and using the three expressions in (12), we have that

$$
\begin{aligned}
\dot{V}_{E 1}= & \varepsilon\left[i_{c}^{d}-\widetilde{I}-i^{d}-\frac{\varepsilon}{R_{c}}-\frac{v^{d}}{R_{c}}-C \frac{\mathrm{d} v^{d}}{\mathrm{~d} t}\right]+\frac{1}{2} \frac{\mathrm{d} L(y)}{\mathrm{d} y} \dot{y} \widetilde{I}^{2} \\
& +\widetilde{I}\left[-\frac{\mathrm{d} L(y)}{\mathrm{d} y} \widetilde{I} \frac{\mathrm{d} y}{\mathrm{~d} t}-\frac{\mathrm{d} L(y)}{\mathrm{d} y} i \frac{\mathrm{d} y}{\mathrm{~d} t}-R \widetilde{I}-R i^{d}\right. \\
& \left.+\varepsilon+v^{d}-L(y) \frac{\mathrm{d} i^{d}}{\mathrm{~d} t}\right] \\
& +\dot{y}\left[\frac{1}{2} \frac{\mathrm{d} L(y))^{2}}{\mathrm{~d} y}+\frac{\mathrm{d} L(y)}{\mathrm{d} y} i^{d} \widetilde{I}+\frac{1}{2} \frac{\mathrm{d} L(y)_{i}}{\mathrm{~d} y} i^{d 2}+m g\right] .
\end{aligned}
$$

Hence, choosing $i_{c}^{d}=\left(v^{d} / R_{c}\right)+i_{c d}^{d}, \quad i_{c d}^{d}=-k_{1} \varepsilon$, $v^{d}=-k_{2} \tilde{I}, i^{d}=\sqrt{2 F^{d} /|(\mathrm{d} L(y) / \mathrm{d} y)|}, F^{d}=F_{d}+F_{s}$, and $F_{d}=$ $k_{3} \dot{y}$ and taking advantage from several natural cancellations, as in (10), we have

$$
\begin{aligned}
\dot{V}_{E 1}= & -\left(\frac{1}{R_{c}}+k_{1}\right) \varepsilon^{2}+\varepsilon\left[-i^{d}-C \frac{\mathrm{d} v^{d}}{\mathrm{~d} t}\right]-\left(R+k_{2}\right) \widetilde{I}^{2} \\
& +\widetilde{I}\left[-R i^{d}-L(y) \frac{\mathrm{d} i^{d}}{\mathrm{~d} t}\right]-k_{3} \dot{y}^{2}-\dot{y} F_{s}+\dot{y}[m g],
\end{aligned}
$$

where $(\mathrm{d} L(y) / \mathrm{d} y) /(|\mathrm{d} L(y) / \mathrm{d} y|)=-1$. Finally, if we choose $F_{s}=k_{4}\left(y-y^{*}\right)+m g$ and $V_{E}=V_{E 1}+(1 / 2) k_{4}\left(y-y^{*}\right)^{2}$, we find

$$
\begin{aligned}
\dot{V}_{E}= & -\left(\frac{1}{R_{c}}+k_{1}\right) \mathcal{\varepsilon}^{2}+\varepsilon\left[-i^{d}-C \frac{\mathrm{d} v^{d}}{\mathrm{~d} t}\right]-\left(R+k_{2}\right) \widetilde{I}^{2} \\
& +\widetilde{I}\left[-R i^{d}-L(y) \frac{\mathrm{d} i^{d}}{\mathrm{~d} t}\right]-k_{3} \dot{y}^{2} .
\end{aligned}
$$

Thus, $F_{s}$ suitably shapes the potential energy of the mechanical subsystem to have a unique minimum at $y=y^{*}$, whereas $F_{d}, i_{c d}^{d}$, and $v^{d}$, represent the damping injection terms, as usual in standard passivity-based control [20].

In Section 4, we will show that several cross terms arising from the rectangular brackets in (15) do not cancel naturally. This means that additional terms must be included in the control law if they are required to be cancelled. Hence, we prefer to dominate these terms instead of feeding back them in order to artificially cancel them. This allows us to design simpler control laws when compared to previous passivitybased approaches [20] where those terms must be online computed and fed back in order to be cancelled artificially. 
In this respect, we stress that it is recognized in the literature that increasing the number of online computations deteriorates performance because this increases numerical errors and the effects of noise. Moreover, we will also show that including PI and PID controllers (instead of proportional controllers as above) is straightforward. Notice that this feature is important to render robust the control scheme. The features described in this paragraph render novel and advantageous our passivity-based approach with respect to that in [20] where the natural cancellations shown in (10) are not exploited.

\section{Main Result}

Our main result is stated in the following proposition.

Proposition 1. Consider the mathematical model in (1)-(5) in a closed loop with the following controller:

$$
\begin{aligned}
u= & \frac{1}{2}\left[1-\operatorname{sign}\left(s_{c}\right)\right], \quad s_{c}=i_{c}-i_{c}^{*}, \\
i_{c}^{*}= & \frac{\bar{v}}{R_{c}}-k_{p 1} e-k_{i 1} \int_{0}^{t} e(r) \mathrm{d} r \\
& -C \alpha_{p}\left(-\frac{\mathrm{d} L(y)}{\mathrm{d} y} i^{*} \dot{y}+v\right)-C \alpha_{i} \tilde{i}, \quad e=v-\bar{v}, \\
\bar{v}= & -\alpha_{p} L(y) \widetilde{i}-\alpha_{i} \int_{0}^{t} \widetilde{i}(r) \mathrm{d} r, \quad \widetilde{i}=i-i^{*}, \\
i^{*}= & \sqrt{\frac{2}{|\mathrm{~d} L(y) / \mathrm{d} y|} F^{*},} \\
F^{*}= & k_{p} h(\widetilde{y})+k_{d} \dot{y}+k_{i} \operatorname{sat}(z), \quad \widetilde{y}=y-y^{*}, \\
z= & \int_{0}^{t}\left[\alpha\left(1+\frac{\beta k_{p}}{k_{i}}\right) h(\tilde{y})+\left(1+\frac{\alpha \beta k_{d}}{k_{i}}\right) \dot{y}\right] \mathrm{d} s,
\end{aligned}
$$

where $y^{*}>0$ is a real constant standing for the desired position, $h(\tilde{y})=\sigma(\tilde{y})$, and sat $(z)=\sigma(z)$, where $\sigma(\cdot)$ is a strictly increasing linear saturation function for some $\left(L^{*}, M\right)$ (see Definition 1). Furthermore, it is also required that function $\sigma(\cdot)$ be continuously differentiable such that

$$
0<\frac{\mathrm{d} \sigma(\varsigma)}{\mathrm{d} \varsigma} \leq 1, \quad \forall \varsigma \in \mathscr{R} .
$$

The closed-loop state evolution is assumed to be constrained to a subset $\mathscr{D} \subset \mathscr{R}^{7}$, where

$$
\begin{array}{r}
\left|\frac{\mathrm{d} L(y)}{\mathrm{d} y}\right|>\gamma_{1}, \\
F^{*}>\gamma_{2}, \\
|\tilde{y}| \leq L^{*},
\end{array}
$$

for some $\gamma_{1}>0$ and $\gamma_{2}>0$. Under these conditions there always exist constant scalars $\alpha, \beta, k_{p 1}, k_{i 1}$, $k_{p}, k_{d}, k_{i}, \alpha_{p}$, and $\alpha_{i}$, such that the closed-loop system has a unique equilibrium point which is asymptotically stable as long as

$$
0<v+L_{c} \frac{\mathrm{d} i_{c}^{*}}{\mathrm{~d} t}<E .
$$

At this equilibrium point, $\tilde{y}=0$.

4.1. Reaching the Sliding Surface. The time derivative of the positive definite and radially unbounded scalar function $V_{c}\left(s_{c}\right)=(1 / 2) s_{c}^{2}$, along the trajectories of [1] is

$$
\begin{aligned}
\dot{V}_{c} & =s_{c} \dot{s}_{c}=s_{c}\left[\frac{\mathrm{d} i_{c}}{\mathrm{~d} t}-\frac{\mathrm{d} i_{c}^{*}}{\mathrm{~d} t}\right] \\
& \left.\leq \frac{\left|s_{c}\right|}{L_{c}}||-v-L_{c} \frac{\mathrm{d} i_{c}^{*}}{\mathrm{~d} t}+\frac{1}{2} E \mid-\frac{1}{2} E\right]<0,
\end{aligned}
$$

where [15] has been used, if $\left|-v-L_{c}\left(\mathrm{~d} i_{c}^{*} / \mathrm{d} t\right)+(1 / 2) E\right|-$ $(1 / 2) E<0$. By considering the two possibilities $-v-$ $L_{c}\left(\mathrm{~d} i_{c}^{*} / \mathrm{d} t\right)+(1 / 2) E>0$ and $-v-L_{c}\left(\mathrm{~d} i_{c}^{*} / \mathrm{d} t\right)+(1 / 2) E<0$, it is not difficult to show that (24) implies (23). From the sliding condition $\dot{s}_{c}=0,[1,22]$, we find that the equivalent control satisfies the following bound:

$$
0<u_{\mathrm{eq}}=\frac{1}{E}\left[v+L_{c} \frac{\mathrm{d} i_{c}^{*}}{\mathrm{~d} t}\right]<1,
$$

which means that the sliding regime is possible. On the contrary, (24) ensure that the sliding surface $s_{c}=i_{c}-i_{c}^{*}=0$ is reached, i.e., $i_{c}=i_{c}^{*}$ is reached. Thus, we only have to study the stability of dynamics (2)-(5) in closed loop with (17)-(20) when evaluated at $i_{c}=i_{c}^{*}$.

4.2. Closed-Loop Dynamics on the Sliding Surface. Using $i_{c}=i_{c}^{*},[16]$ in [2], and adding and subtracting the terms $i^{*}$, $\left(1+C \alpha_{p} R\right) \sqrt{2 m g /\left(\left|\mathrm{d} L\left(y^{*}\right) / \mathrm{d} y\right|\right)}, C \dot{\bar{v}}$, we find

$$
\begin{aligned}
C \dot{e}= & -\left(\frac{1}{R_{c}}+k_{p 1}\right) e-\tilde{i}-\left(1+C \alpha_{p} R\right)\left[i^{*}-\sqrt{\frac{2 m g}{\left|\mathrm{~d} L\left(y^{*}\right) / \mathrm{d} y\right|}}\right] \\
& -k_{i 1} \xi-C \alpha_{p} L(y) \frac{\mathrm{d} i^{*}}{\mathrm{~d} t}-C \alpha_{p} R \tilde{i},
\end{aligned}
$$

$$
\xi=\int_{0}^{t} e(r) \mathrm{d} r+\frac{1+C \alpha_{p} R}{k_{i 1}} \sqrt{\frac{2 m g}{\left|\mathrm{~d} L\left(y^{*}\right) / \mathrm{d} y\right|}},
$$

where

$$
\frac{\mathrm{d} L\left(y^{*}\right)}{\mathrm{d} y}=\left.\frac{\mathrm{d} L(y)}{\mathrm{d} y}\right|_{y=y^{*}} .
$$

On the other hand, adding and subtracting the terms $i^{*}$, $\bar{v}, L(y)\left(\mathrm{d} i^{*} / \mathrm{d} t\right), R \sqrt{2 m g /\left(\left|\mathrm{d} L\left(y^{*}\right) / \mathrm{d} y\right|\right)}, L(y)\left(\mathrm{d} i^{*} / \mathrm{d} t\right)$, and $(\mathrm{d} L(y) / \mathrm{d} y) i^{*} \dot{y}$, in [3], and replacing [17], we obtain 


$$
\begin{aligned}
L(y) \frac{\mathrm{d} \widetilde{i}}{\mathrm{~d} t}= & e-\left(R+\alpha_{p} L(y)\right) \tilde{i}-\alpha_{i} z_{1}-R\left[i^{*}-\sqrt{\mathrm{d} y} \tilde{i} \dot{y}-\frac{\mathrm{d} L(y)}{\mathrm{d} y} i^{*} \dot{y}-L(y) \frac{\mathrm{d} i^{*}}{\mathrm{~d} t},\right. \\
& -\frac{\mathrm{d} L(y)}{z_{1}=} \int_{0}^{t} \tilde{i}(r) \mathrm{d} r+\frac{R}{\alpha_{i}} \sqrt{\frac{2 m g}{\left|\mathrm{~d} L\left(y^{*}\right) / \mathrm{d} y\right|}}
\end{aligned}
$$

where

$$
\begin{aligned}
\frac{\mathrm{d} i^{*}}{\mathrm{~d} t}= & \left(\frac{2 F^{*}}{|\mathrm{~d} L(y) / \mathrm{d} y|}\right)^{-1 / 2} \times\left\{\left(\frac{\mathrm{d}}{\mathrm{d} y}\left|\frac{\mathrm{d} L(y)}{\mathrm{d} y}\right|^{-1}\right) \dot{y} \times\left[k_{p} h(\tilde{y})\right.\right. \\
& \left.+k_{d} \dot{y}+k_{i} s(z)+m g\right] \\
& \left.+\left|\frac{\mathrm{d} L(y)}{\mathrm{d} y}\right|^{-1}\left[k_{p} \frac{\mathrm{d} h(\tilde{y})}{\mathrm{d} \tilde{y}} \dot{y}+k_{d} \ddot{y}+k_{i} \frac{\mathrm{dsat}(z)}{\mathrm{d} z} \dot{z}\right]\right\},
\end{aligned}
$$

$$
s(z)=\operatorname{sat}(z)-\frac{1}{k_{i}} m g .
$$

Finally, adding and subtracting the terms $1 / 2(\mathrm{~d} L(y) /$ $\mathrm{d} y) i^{*} i,(1 / 2)(\mathrm{d} L(y) / \mathrm{d} y) i^{*} \widetilde{i}$, and $(1 / 2)(\mathrm{d} L(y) / \mathrm{d} y) i^{* 2}$, and replacing $i^{*}$ and $F^{*}$ from [18], the expression in [4] becomes

$$
m \ddot{y}=\frac{1}{2} \frac{\mathrm{d} L(y)_{\tilde{i}^{2}}}{\mathrm{~d} y}+\frac{\mathrm{d} L(y)}{\mathrm{d} y} \widetilde{i}^{*}-k_{p} h(\widetilde{y})-k_{d} \dot{y}-k_{i} s(z) .
$$

The closed-loop dynamics is given by (26)-(33) and (20). Equilibria of this dynamics are found as follows. From the state equation $\dot{\tilde{y}}=\dot{y}=0$, it is concluded that $\dot{y}=0$ at the equilibrium point. Using this result in $\dot{z}=0$ (from (20)) yields $\tilde{y}=0$. From $\dot{z}_{1}=0$ (see (30)), we find $\widetilde{i}=0$. Then, from $m \ddot{y}=0$, in (33), we find $z=\left(1 / k_{i}\right) m g$ if

$$
L^{*}>\frac{1}{k_{i}} m g \text {. }
$$

Using the above results in (19) yields $i^{*}=\sqrt{2 m g /\left(\left|\mathrm{d} L\left(y^{*}\right) / \mathrm{d} y\right|\right)}$ and from $\dot{\xi}=0$ in (27), we find that $e=0$. From (31), we have that $\mathrm{d} i^{*} / \mathrm{d} t=0$. Using $\dot{e}=0$ in (26), we have that $\xi=0$ at the equilibrium point. Hence, from (29), we find that $z_{1}=0$.

This means that the only equilibrium point of the closedloop dynamics is $\zeta=\left[\dot{y}, \tilde{y}, z-\left(1 / k_{i}\right) m g, \tilde{i}, z_{1}, e, \xi\right]^{T}=$ $[0,0,0,0,0,0,0]^{T}$. Notice that this closed-loop dynamics is autonomous because it can be written as $\dot{\zeta}=f(\zeta)$ for some nonlinear $f(\cdot) \in \mathscr{R}^{7}$.

4.3. Stability Analysis. The closed-loop dynamics (26)-(33) and (20) can be rewritten as follows:

$$
\begin{aligned}
& C \dot{e}=\mathscr{I}_{c}-\tilde{i}-\left(\frac{1}{R_{c}}+k_{p 1}\right) e, \\
& L(y) \frac{\widetilde{\mathrm{d}}}{\mathrm{d} t}=-\left(R+\alpha_{p} L(y)\right) \tilde{i}-\frac{\mathrm{d} L(y)}{\mathrm{d} y} \widetilde{i} \dot{y}+\Upsilon, \\
& m \ddot{y}=\frac{1}{2} \frac{\mathrm{d} L(y)}{\mathrm{d} y} \tilde{i}^{2}-\mathscr{G}, \\
& \dot{z}_{1}=\widetilde{i}, \quad \dot{\xi}=e, \\
& \dot{z}=\alpha\left(1+\frac{\beta k_{p}}{k_{i}}\right) h(\tilde{y})+\left(1+\frac{\alpha \beta k_{d}}{k_{i}}\right) \dot{y}, \\
& \mathscr{I}_{c}=-\left(1+C \alpha_{p} R\right)\left[i^{*}-\sqrt{\frac{2 m g}{\left|\mathrm{~d} L\left(y^{*}\right) / \mathrm{d} y\right|}}\right]-k_{i 1} \xi \\
& -C \alpha_{p} L(y) \frac{\mathrm{d} i^{*}}{\mathrm{~d} t}-C \alpha_{p} R \tilde{i}, \\
& \mathscr{G}=-\frac{\mathrm{d} L(y)}{\mathrm{d} y} \tilde{i i}^{*}+k_{p} h(\widetilde{y})+k_{d} \dot{y}+k_{i} s(z), \\
& \Upsilon=e-R\left(i^{*}-\sqrt{\frac{2 m g}{\left|\mathrm{~d} L\left(y^{*}\right) / \mathrm{d} y\right|}}\right)-\frac{\mathrm{d} L(y)}{\mathrm{d} y} i^{*} \dot{y} \\
& -L(y) \frac{\mathrm{d} i^{*}}{\mathrm{~d} t}-\alpha_{i} z_{1} .
\end{aligned}
$$

Notice that (35)-(38) are almost identical to the openloop dynamics in (7) if we replace $y, \dot{y}, i, v, G, i_{c}$ by $\tilde{y}, \dot{y}, \tilde{i}, \Upsilon, \mathscr{G}, \mathscr{I}_{c}$. One important difference is that the resistances $R_{c}$ and $R$ in (7) have been enlarged to $\left(1 / R_{c}\right)+k_{p 1}$ and $R+\alpha_{p} L(y)$ in (35) and (36), respectively. Moreover, we can see that suitable damping can be introduced thanks to term $k_{d} \dot{y}$ in the definition of $\mathscr{G}$. Another important difference is the three new equations in (38) which represent the integral terms of the PI electric current controller, the PI controller of voltage at the capacitor, and the PID position controller, which are intended to compensate for the effects of the gravity term $m g$.

These observations motivate the use of the following "energy" storage function for the closed-loop dynamics:

$$
\begin{aligned}
W\left(\dot{y}, \widetilde{y}, z-\frac{m g}{k_{i}}, \widetilde{i}, z_{1}, e, \xi\right)= & \frac{1}{2} C e^{2}+\frac{1}{2} k_{i 1} \xi^{2}+\frac{1}{2} L(y) \widetilde{i}^{2} \\
& +\frac{1}{2} \alpha_{i} z_{1}^{2}+V_{m}\left(\dot{y}, \widetilde{y}, z-\frac{m g}{k_{i}}\right),
\end{aligned}
$$

where

$$
\begin{aligned}
V_{m}\left(\tilde{y}, \dot{y}, z-\frac{m g}{k_{i}}\right)= & \frac{1}{2} m \dot{y}^{2}+\alpha m h(\tilde{y}) \dot{y}+\alpha k_{d} \int_{0}^{\tilde{y}} h(r) \mathrm{d} r \\
& +k_{p} \int_{0}^{\tilde{y}} h(r) \mathrm{d} r+k_{i} \int_{m g / k_{i}}^{z} s(r) \mathrm{d} r \\
& +\alpha \beta m s(z) \dot{y} .
\end{aligned}
$$


We stress that function $V_{m}\left(\dot{y}, \tilde{y}, z-m g / k_{i}\right)$ defined in (40) is very similar to the function $V\left(\widetilde{q}, \dot{q}, z-\left(k_{i}^{\prime}\right)^{-1} g\left(q_{d}\right)\right)$ analyzed in [30]. Thus, conditions to ensure that $V_{m}\left(\dot{y}, \tilde{y}, z-m g / k_{i}\right)$ is positive definite and radially unbounded are the same as those introduced in [30]. For the sake of completeness of this work, these conditions are presented in Appendix A as (A.1), (A.2), and (A.5) and $k_{p}>0, \quad \alpha>0, \quad$ and $\beta>0$. Thus, the function $W\left(\dot{y}, \tilde{y}, z-m g / k_{i}, \widetilde{i}, z_{1}, e, \xi\right)$ qualifies as a Lyapunov function candidate because it is positive definite and radially unbounded if $k_{i 1}>0$ and $\alpha_{i}>0$.

The first two terms in $W$ represent the electric energy stored in the converter's capacitor and "energy" stored in the integral term of the PI voltage controller. The third and fourth terms represent the magnetic energy stored in the electrical system and the "energy" stored in the integral term of the PI electric current controller. On the other hand, function $V_{m}$ includes the kinetic energy and the closed-loop "potential energy"

$$
\mathscr{P}(\tilde{y})=k_{p} \int_{0}^{\tilde{y}} h(r) \mathrm{d} r,
$$

as well as the "energy" stored in the integral of position through an integral of $s(\cdot)$. The cross terms $\alpha m h(\widetilde{y}) \dot{y}$ and $\alpha \beta m s(z) \dot{y}$ are required to provide $\dot{W}$ with negative quadratic terms in both $h(\widetilde{y})$ and $s(z)$. In this respect, it is easy to verify that

$$
\begin{aligned}
\frac{\mathrm{d}}{\mathrm{d} t}\left(\frac{1}{2} L(y) \widetilde{i}^{2}+\frac{1}{2} m \dot{y}^{2}+\mathscr{P}(\tilde{y})\right)= & -\left(R+\alpha_{p} L(y)\right) \widetilde{i}^{2}-k_{d} \dot{y}^{2} \\
& +\widetilde{i} \Upsilon+\frac{\mathrm{d} L(y)}{\mathrm{d} y} \tilde{y} \tilde{i}^{*}-k_{i} s(z) \dot{y} .
\end{aligned}
$$

Since $\Upsilon$ depends on both $h(\widetilde{y})$ and $s(z)$, negative quadratic terms on both $h(\tilde{y})$ and $s(z)$ are required to dominate some cross terms in both $h(\widetilde{i})$ and $\widetilde{i}$ and $s(z)$ and $\tilde{i}$. This is the reason for the cross terms $\alpha m h(\tilde{y}) \dot{y}$ and $\alpha \beta m s$ $(z) \dot{y}$ (the quadratic term $-\left(R+\alpha_{p} L(y)\right) \tilde{i}^{2}$ already exists). The integral term $\alpha k_{d} \int_{0}^{y} h(r) \mathrm{d} r$ is intended to cancel an undesired cross term appearing in the time derivative of $\alpha m h(\widetilde{y}) \dot{y}$. Notice that term $\widetilde{i} e$, arising from the product $\tilde{i} Y$ above, cancels with term $-e \tilde{i}$, arising from $(\mathrm{d} / \mathrm{d} t)\left(C e^{2} / 2\right)$. Moreover, some third order terms involving $i^{*}$ appear from $\widetilde{i}$. These terms can be dominated by quadratic negative terms in $\dot{y}$ and $\tilde{i}$ because $|h(\tilde{y})|$ and $|s(z)|$ are bounded by finite constants. This is the reason to employ a PID position controller with saturated proportional and integral actions.

After some straightforward natural cancellations (i.e., not requiring to use additional terms in the control law to achieve them), which include the closed-loop equivalents of (10) (see Remark 6), we find that the time derivative of $W$ along the trajectories of the closed-loop systems (35)-(38) is given as follows:

$$
\begin{aligned}
\dot{W}= & -\left(\frac{1}{R_{c}}+k_{p 1}\right) e^{2}-\left(R+\alpha_{p} L(y)-\frac{\alpha}{2} \frac{\mathrm{d} L(y)}{\mathrm{d} y} h(\widetilde{y})-\frac{\alpha \beta}{2} \frac{\mathrm{d} L(y)}{\mathrm{d} y} s(z)\right) \tilde{i}^{2} \\
& -\left[k_{d}-\alpha m \frac{\mathrm{d} h(\widetilde{y})}{\mathrm{d} \widetilde{y}}-\alpha \beta m \frac{\mathrm{d} s(z)}{\mathrm{d} z}\left(1+\frac{\alpha \beta k_{d}}{k_{i}}\right)\right] \dot{y}^{2}-\alpha k_{p} h^{2}(\widetilde{y})-\alpha \beta k_{i} s^{2}(z) \\
& -\left[e\left(1+C \alpha_{p} R\right)+R \widetilde{i}\right]\left(i^{*}-\sqrt{\frac{m g}{\left|\mathrm{~d} L\left(y^{*}\right) / \mathrm{d} y\right|}}\right)+\alpha \frac{\mathrm{d} L(y)}{\mathrm{d} y} h(\widetilde{y}) \tilde{i} \tilde{i}^{*}+\alpha^{2} \beta m \frac{\mathrm{d} s(z)}{\mathrm{d} z}\left(1+\frac{\beta k_{p}}{k_{i}}\right) h(\widetilde{y}) \dot{y} \\
& +\alpha \beta \frac{\mathrm{d} L(y)}{\mathrm{d} y} s(z) \tilde{i i}^{*}-C \alpha_{p} \operatorname{Re} \tilde{i}-L(y) \tilde{i} \frac{\mathrm{d} i^{*}}{\mathrm{~d} t}-C \alpha_{p} e L(y) \frac{\mathrm{d} i^{*}}{\mathrm{~d} t} .
\end{aligned}
$$

Taking into account (19), (31), and Appendix B, it is found that $\dot{W}$ can be upper bounded as follows:

$$
\begin{aligned}
\dot{W} \leq & -x^{T} Q x-\alpha_{p 2} L(y) \widetilde{i}^{2}+\left.\frac{1}{2 m} k_{r r} k_{d} k_{\sigma} \widetilde{i}\right|^{3}+\frac{C \alpha_{p}}{2 m} k_{r r} k_{d} k_{\sigma}|e||\widetilde{i}|^{2}+\frac{C \alpha_{p}}{2 m} k_{r r} k_{d} k_{\sigma} k_{\delta}|e||\widetilde{i}||\dot{y}| \\
& -\alpha_{p 4} L(y) \tilde{i}^{2}-k_{p 3} e^{2}-k_{d 4} \dot{y}^{2}+\frac{1}{2 m} k_{r r} k_{d} k_{\sigma} k_{\delta} \tilde{i}^{2}|\dot{y}|-\alpha_{p 3} L(y) \tilde{i}^{2}+\left[C \alpha_{p} k_{r} k_{d}+\left(1+C \alpha_{p} R\right) k_{t}\right]|e| \dot{y}^{2}-k_{d 3} \dot{y}^{2} \\
& +\left(k_{r} k_{d}+R k_{t}\right)|| i \mid \dot{y}^{2}-k_{d 2} \dot{y}^{2}, \\
x^{T}= & {[|\dot{y}|,|h(\tilde{y})|,|s(z)|,|\widetilde{i}|,|e|], }
\end{aligned}
$$


where $k_{d 1}, k_{d 2}, k_{d 3}, k_{d 4}, \alpha_{p 1}, \alpha_{p 2}, \alpha_{p 3}, \alpha_{p 4}, k_{p 2}$, and $k_{p 3}$ are positive constant scalars such that $k_{d 1}+k_{d 2}+k_{d 3}+k_{d 4}=k_{d}$, $\alpha_{p 1}+\alpha_{p 2}+\alpha_{p 3}+\alpha_{p 4}=\alpha_{p}$, and $k_{p 2}+k_{p 3}=k_{p 1}$. The entries of matrix $Q$ are defined as follows:

$$
\begin{aligned}
& Q_{11}=k_{d 1}-\alpha m-\alpha \beta m\left(1+\frac{\alpha \beta k_{d}}{k_{i}}\right), \\
& Q_{22}=\alpha k_{p}, \\
& Q_{33}=\alpha \beta k_{i} \\
& Q_{44}=R+\alpha_{p 1} L(y)-\frac{\alpha}{2} k_{\sigma} M-\alpha \beta k_{\sigma} M-\frac{1}{m} k_{r r} k_{d} k_{\sigma} I^{*}(0), \\
& Q_{55}=\frac{1}{R_{c}}+k_{p 2} \\
& Q_{12}=Q_{21}=-\frac{\alpha^{2} \beta m}{2}\left(1+\frac{\beta k_{p}}{k_{i}}\right) \\
& Q_{31}=Q_{13}=Q_{23}=Q_{32}=0 \text {, } \\
& Q_{14}=Q_{41}=-\frac{k_{r} k_{p} M}{2}-k_{r} k_{i} M-\frac{k_{r} m g}{2}-\frac{k_{r r} k_{p}}{2}-\frac{k_{r r} k_{d}^{2}}{2 m}-\frac{R k^{*}}{2} \\
& -\frac{k_{i} k_{r r}}{2}\left(1+\frac{\alpha \beta k_{d}}{k_{i}}\right)-\frac{\alpha k_{\sigma} k_{\delta} M}{2}-\alpha \beta M k_{\delta} k_{\sigma}-\frac{3 R k_{t} M}{2}, \\
& Q_{42}=Q_{24}=-\frac{k_{r r} k_{d} k_{p}}{2 m}-\frac{\alpha k_{i} k_{r r}}{2}\left(1+\frac{\beta k_{p}}{k_{i}}\right)-\frac{\alpha k_{\sigma} I^{*}(0)}{2}-\frac{R k^{*}}{2}, \\
& Q_{43}=Q_{34}=-\frac{k_{r r} k_{d} k_{i}}{2 m}-\frac{\alpha \beta k_{\sigma} I^{*}(0)}{2}-\frac{R k^{*}}{2}, \\
& Q_{15}=Q_{51}=-\frac{\left(1+C \alpha_{p} R\right) k^{*}}{2}-\frac{3\left(1+C \alpha_{p} R\right) k_{t} M}{2}-\frac{C \alpha_{p} k_{r} k_{p} M}{2} \\
& -C \alpha_{p} k_{r} k_{i} M-C \alpha_{p} \frac{k_{r} m g}{2}-C \alpha_{p} \frac{k_{r r} k_{p}}{2}-C \alpha_{p} \frac{k_{r r} k_{d}^{2}}{2 m}-C \alpha_{p} \frac{k_{i} k_{r r}}{2}\left(1+\frac{\alpha \beta k_{d}}{k_{i}}\right), \\
& Q_{52}=Q_{25}=-\frac{\left(1+C \alpha_{p} R\right) k^{*}}{2}-C \alpha_{p} \frac{k_{r r} k_{d} k_{p}}{2 m}-C \alpha_{p} \frac{\alpha k_{i} k_{r r}}{2}\left(1+\frac{\beta k_{p}}{k_{i}}\right) \\
& Q_{53}=Q_{35}=-\frac{\left(1+C \alpha_{p} R\right) k^{*}}{2}-C \alpha_{p} \frac{k_{r r} k_{d} k_{i}}{2 m}, \\
& Q_{45}=Q_{54}=-\frac{1}{2} C \alpha_{p} R-\frac{C \alpha_{p}}{2 m} k_{r r} k_{d} k_{\sigma} I^{*}(0),
\end{aligned}
$$

where

$$
\begin{aligned}
& k_{r}=\max \left\{\left.L(y)\left(\frac{2 F^{*}}{|\mathrm{~d} L(y) / \mathrm{d} y|}\right)^{-1 / 2}\left|\frac{d}{d y}\right| \frac{\mathrm{d} L(y)}{\mathrm{d} y}\right|^{-1} \mid\right\}, \\
& k_{r r}=\max \left\{L(y)\left(\frac{2 F^{*}}{|\mathrm{~d} L(y) / \mathrm{d} y|}\right)^{-1 / 2}\left|\frac{\mathrm{d} L(y)}{\mathrm{d} y}\right|^{-1}\right\},
\end{aligned}
$$


and constants $k_{\sigma}, k_{\delta}, k^{*}, k_{t}$, and $I^{*}(0)$ are defined in Appendix $\mathrm{B}$.

Notice that the four leading principal minors of matrix $Q$ can always be rendered positive definite by suitable selection of the controller gains $k_{p 1}, k_{d 1}, k_{p}, k_{i}$, and $\alpha_{p 1}$, and hence, $\lambda_{\min }(Q)>0$. Furthermore, all of the expressions in the seven rows in (45) can be rendered negative, at least locally, using suitable gains $k_{d 2}, k_{d 3}, k_{d 4}, \alpha_{p 2}, \alpha_{p 3}, \alpha_{p 4}, k_{p 2}$, and $k_{p 3}$. Hence, it is concluded that $\dot{W} \leq 0$ for all $\zeta \in \mathscr{D}$, where $\mathscr{D}$ is a subset of $\mathscr{R}^{7}$ containing the origin. Thus, stability of the origin is concluded. Since the closed-loop system is autonomous, the LaSalle invariance principle is invoked to prove asymptotic stability. This completes the proof of Proposition 1.

Conditions for this stability result are summarized by (A.1), (A.2), and (A.5), in Appendix A, $k_{p}>0, \alpha>0, \beta>0$, $k_{i 1}>0$, and $\alpha_{i}>0$, (34), the four leading principal minors of matrix $Q$ defined in (45) are positive, and $k_{d 2}, k_{d 3}, k_{d 4}, \alpha_{p 2}$, $\alpha_{p 3}, \alpha_{p 4}, k_{p 2}$, and $k_{p 3}$ are chosen such that the seven rows in (45) are rendered negative. These stability conditions constitute explicit tuning guide lines.

Remark 1. Notice that the ith leading principal minor of matrix $Q$ can be rendered positive by choosing large enough $Q_{i i}$ entry of matrix $Q$ and choosing small some constants such as $\alpha>0$ and $\beta>0$. In this respect, it is important to stress that each one of the $Q_{i i}$ entries depend on a controller gain. Thus, once the $i$ th leading principal minor is rendered positive by choosing a suitable controller gain, the $(i+1)$ th leading principal minor can be rendered positive by suitably enlarging the entry $Q_{(i+1)(i+1)}$ of matrix $Q$ and so on. Moreover, these ideas suggest that an intuitive try-and-error tuning procedure can also be derived; choose larger controller gains in the $Q_{i i}$ entries as $i$ is larger.

Remark 2. Notice that the system evolves by itself as long as the sliding surface is not reached. However, it is proven in standard books on sliding mode control that the system reaches the sliding surface in a finite time which depends on the initial conditions. Once the sliding surface is reached, the system evolves on the sliding surface starting from the state values that it has at the time where the sliding surface is reached. Thus, if initial conditions are close to the desired equilibrium point, then asymptotic convergence to the desired equilibrium point is ensured by Proposition 1 .

On the other hand, limits in currents $i_{c}$ and $i$ are imposed by the circuit parameters and the applied voltage. With the problem at hand, limits on these currents are imposed by the DC power supply $E$ which is manipulated through the variable $u$ representing the on-off state of transistor $Q$. In Section 5, we explain how the variables $u$ and $E$ affect the system performance. Finally, saturation of the internal PI's only might occur if the state variables are allowed to take large values. Since our stability result is local, this prohibits the state to take such large values.

Remark 3. In Figure 3, we present a block diagram of the control scheme in control scheme in Proposition 1. Notice that this controller is made up of three main loops: (1) a PI controller for voltage at the DC/DC Buck power converter output (at the capacitor terminals), (2) a PI controller for electric current through the electromagnet, and (3) a nonlinear PID (NPID) controller for the ball position. Thus, our proposal contains the fundamental components in industrial applications and, hence, it is expected to be robust with respect to parametric uncertainties and external disturbances. Furthermore, another internal loop is provided to control electric current through the inductance of the DC/ DC Buck power converter. This loop is driven by a sliding mode controller which constitutes one common technique to control power electronic devices in practice.

Remark 4. It is stressed that we introduce the factor $L(y)$ as a part of the proportional gain in (18) in order to ensure to be constant the term that is added to the integral term in (27). This is a necessary step for the integral action of the PI controller of voltage at the capacitor. In this respect, the last two terms in (17) are included in order to cancel some terms arising in (26) because of the fact that $-C \dot{\bar{v}}$ must be added and subtracted to complete (26).

Remark 5. Notice that the region where the result is valid can be enlarged by including the terms $-k_{q} \widetilde{i} \dot{y}^{2}-k_{f} \widetilde{i} \widetilde{i} \mid-$ $k_{h} \widetilde{i}|e|$ in (18) and the terms $-k_{m} e \dot{y}^{2}-k_{n} e \widetilde{i} \mid$ in (17), for some positive constants $k_{q}, k_{f}, k_{h}, k_{m}$, and $k_{n}$, to proceed as in [30] to dominate the positive terms in the five rows of (45). However, we have decided not to include the above cited terms because of several reasons. (1) The stability result would still remain to be local, as usual in magnetic levitation systems. (2) Including the above terms in (18) would result in additional complex terms that should be cancelled using the definition of $i_{c}^{*}$ in (17). (3) In order to maintain the simple control law, we have decided not to proceed including the terms $-k_{q} \widetilde{i} \dot{y}^{2}-k_{f} \tilde{i} \tilde{i}\left|-k_{h} \tilde{i}\right| e \mid$ in (18) which renders useless to include $-k_{m} e \dot{y}^{2}-k_{n} e|\tilde{i}|$ in (17). Thus, proposing a simple and robust control law performing well is one important objective of our proposal.

Remark 6. The novel passivity-based approach that is employed in this paper has the following properties:

(i) Several terms cancel naturally. This means that they cancel without requiring to compute and feedback them. This property is a direct consequence of the fact that the closed-loop dynamics (35)-(38) is almost identical to the open-loop model in (6). Recall that the open-loop model was proven in Section 3 to be output strictly passive, and the existence of several natural cancellations is instrumental for this. This property is opposite to what happens in exact feedback linearization approaches where the plant undesired terms are online computed and fed back in order to force their cancellation.

The natural cancellation of terms that we refer to in this item is the same that is referred to before (44), which includes $(1 / 2)(\mathrm{d} L(y) / \mathrm{d} y) \tilde{i}^{2} \dot{y}-(\mathrm{d} L(y) / \mathrm{d} y) \widetilde{i}^{2}$ $\dot{y}-(\mathrm{d} L(y) / \mathrm{d} y) \widetilde{i i}^{*} \dot{y}$ belonging to $(\mathrm{d} / \mathrm{d} t)((1 / 2) L(y)$ $\left.\widetilde{i}^{2}\right)$ and $(1 / 2)(\mathrm{d} L(y) / \mathrm{d} y) \dot{i}^{2} \dot{y}+(\mathrm{d} L(y) / \mathrm{d} y) \widetilde{i}^{*} \dot{y}$ belonging to $(\mathrm{d} / \mathrm{d} t)\left((1 / 2) m \dot{y}^{2}\right)$, as well as the 


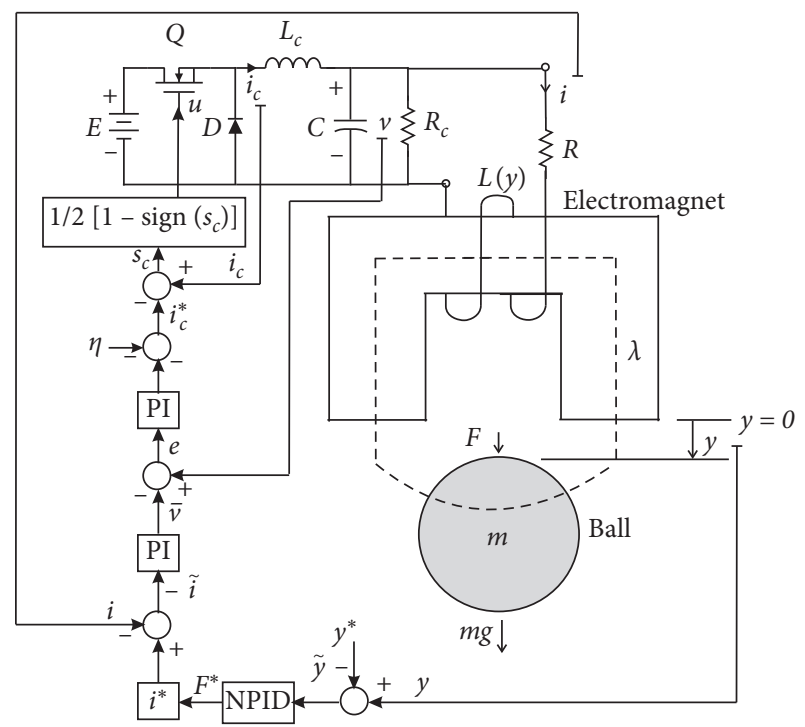

Figure 3: Control scheme in Proposition 1, where $\eta=-\left(\bar{v} / R_{c}\right)+$ $C \alpha_{p}\left(-(\mathrm{d} L(y) / \mathrm{d} y) i^{*} \dot{y}+v\right)+C \alpha_{i} \tilde{i}$.

cancellation of $-e \tilde{i}$ belonging to $(\mathrm{d} / \mathrm{d} t)\left((1 / 2) C e^{2}\right)$ and $\widetilde{e i}$ belonging to $(\mathrm{d} / \mathrm{d} t)\left((1 / 2) L(y) \widetilde{i}^{2}\right)$.

These natural term cancellations are very useful to obtain a simple control law. As stated above, other control approaches require to use additional terms in the control law in order to cancel these terms.

(ii) A nested-loop passivity-based control approach is exploited in [20]. This means that the electric current error is first proven to converge exponentially to zero, and this allows to use this variable as a vanishing perturbation for the mechanical subsystem. This, however, requires the online computation of either the time derivative of the desired force or the time derivative of the desired electric current. Instead of that we use an approach which is similar to what was called in [20] passivity-based control with total energy shaping. Although the latter approach has been disregarded in [20] by arguing that it results in more complex controllers, we prove the opposite in the present paper. This is one important novelty of our approach.

(iii) The previous features of our approach allow (1) to straightforwardly include PI internal loops and an external PID loop, which are important to improve the robustness properties of the control scheme and (2) to avoid the requirement on the exact knowledge of the electric resistance of the electromagnet, which is a parameter that changes during normal operation conditions.

Remark 7. The present work is inspired by [16] in the sense that some PI and PID loops are included for both the electrical and the mechanical dynamics of the electromechanical system, and a sliding mode controller is devoted to control electric current through the inductor of the DC/DC Buck converter, see Figure 4. However, the control scheme in [16] is designed for unidirectional control of velocity in a brushed DC motor, whereas the controller in the present work contains several refinements that extend work in [16] to control position in a magnetic levitation system, a highly nonlinear system. These refinements include (1) a clever selection of a nonlinear PID position controller, (2) a clever selection of internal PI controllers, and (3) proposing a suitable Lyapunov function for stability analysis. We stress that, aside from the sliding mode controller, the closed-loop system in [16] is linear, whereas both plant and controller are nonlinear in the present work.

\section{Simulation Results}

In this section, we present a numerical example to give some insight on the achievable performance when the controller in Proposition 1 is employed. To this aim, we use the numerical values of the magnetic levitation system that has been tested experimentally in [23]. In that work, the electromagnet inductance is modeled as follows:

$$
L(y)=k_{0}+\frac{k}{1+(y / a)},
$$

where $\quad k_{0}=36.3 \times 10^{-3} \mathrm{H}, \quad k=3.5 \times 10^{-3} \mathrm{H}, \quad$ and $a=5.2 \times 10^{-3} \mathrm{~m}$, and the remaining parameters are $R=2.72 \mathrm{Ohm}, m=0.018 \mathrm{~kg}$, and $g=9.81 \mathrm{~m} / \mathrm{s}^{2}$. The practical range of input voltages is $[0,+12] \mathrm{V}$ and the range of electric current through the electromagnet is $[0,+3] \mathrm{A}$. The Buck DC/DC power electronic system parameters were chosen as $L_{c}=0.686 \mathrm{H}, \quad C=114.4 \times 10^{-6} \mathrm{~F}, \quad$ and $R_{c}=28.5 \mathrm{Ohm}$. We also use $E=12 \mathrm{~V}$ if $2 \mathrm{~s}<t<3 \mathrm{~s}$ and $E=50 \mathrm{~V}$, otherwise in order to study the response when disturbances appear in the DC power supply. These parameters are similar to the experimental values reported in [16].

The controller gains were chosen to be $k_{p}=8, k_{d}=1$, $k_{i}=2, \alpha_{p}=470, \alpha_{i}=1000, \alpha=64, \beta=1, k_{p 1}=6000$, and $k_{i 1}=18000$. Inspired by [28], we used the saturation function:

$$
\sigma(x)= \begin{cases}-L^{*}+\left(M-L^{*}\right) \tanh \left(\frac{x+L^{*}}{M-L^{*}}\right), & \text { if } x<-L^{*}, \\ x, & \text { if }|x| \leq L^{*}, \\ L^{*}+\left(M-L^{*}\right) \tanh \left(\frac{x-L^{*}}{M-L^{*}}\right), & \text { if } x>L^{*},\end{cases}
$$

where $M=0.51$ and $L^{*}=0.5$. The initial conditions were set as follows $y(0)=0.006 \mathrm{~m}, \dot{y}(0)=0, \quad i(0)=1.56 \mathrm{~A}$, $v(0)=4.2 \mathrm{~V}, \quad i_{c}(0)=1.65 \mathrm{~A}, \quad \int_{-\infty}^{0} \tilde{i} \mathrm{~d} t=-4.24362 \times 10^{-3}$, $\int_{-\infty}^{0} e \mathrm{~d} t=0.022$, andn $z(0)=0.0885$. The desired position $y^{*}$, in meters, was chosen as follows: 


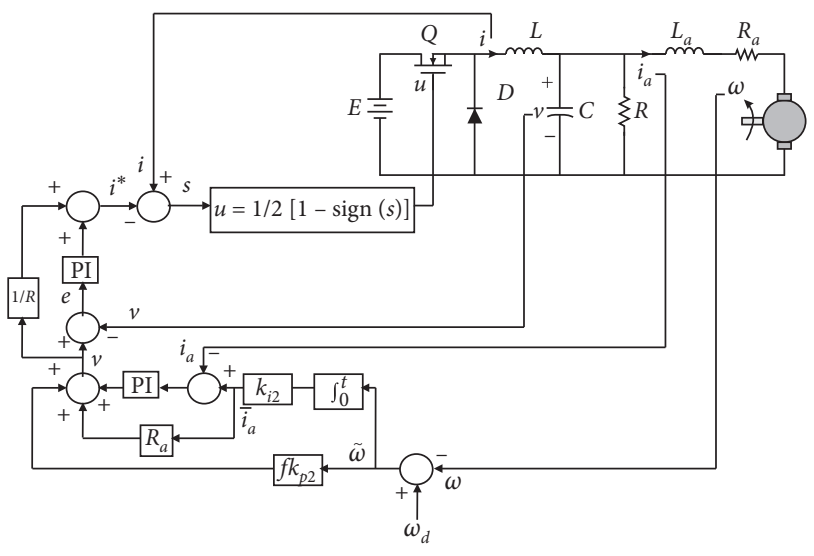

FIgURE 4: Block diagram of the controller in [16].

$$
y^{*}= \begin{cases}0.006, & 0 \leq t<1 \\ 0.008, & 1 \leq t<4 \\ 0.006, & 4 \leq t<5.5 \\ 0.004, & 5.5 \leq t\end{cases}
$$

In Figure 5, we can see that the actual ball position $y$ reaches its desired value $y^{*}$ in the steady state. Notice that the settling time is about $0.5 \mathrm{~s}$. We observe that the position response is very damped. In this respect, we have performed several additional simulations which make us to conclude that the term $\left(1+\alpha \beta\left(k_{d} / k_{i}\right)\right) \dot{y}$ appearing in (20) is responsible for such a damped response.

In Figures 6 and 7, we verify that electric current through the electromagnet $i$ and voltage at the electromagnet terminals $v$ remain within $[0,+3] \mathrm{A}$ and $[0,+12] \mathrm{V}$, respectively, the actual ranges of values reported experimentally in [23].

In Figure 8, we present electric current through the converter inductor which also remains within the range $[0,+3]$ A. Finally, in Figure 9, we verify that evolution of all the above signals is achieved by applying, at the transistor input, a switching signal only taking the discrete values 1 or 0 . Notice that, despite this hard switching signal, electric current through the electromagnet is smooth enough, see Figure 6. Moreover, this is achieved despite voltage at the electromagnet terminals has a small ripple, see Figure 7.

We also observe in Figure 5 that the step changes in the DC power supply, i.e., $E$, appearing at $t=2 \mathrm{~s}$ and $t=3 \mathrm{~s}$ have an almost imperceptible effect in the ball position. Moreover, the effects of these changes are more noticeable in Figures 7 and 8 as a simple change in ripple of both voltage at the electromagnet terminals and electric current through the converter inductor. The effects of these step changes are very small thanks to the employment of a sliding mode controller for electric current through the converter inductor and this is the reason why we employ sliding mode control in our approach.

Finally, we stress that the control signal $u$ is constrained to only take the discrete values 0 or 1 and all of the system evolution must be controlled while $u$ takes these discrete values. However, the designer has an additional degree-of-

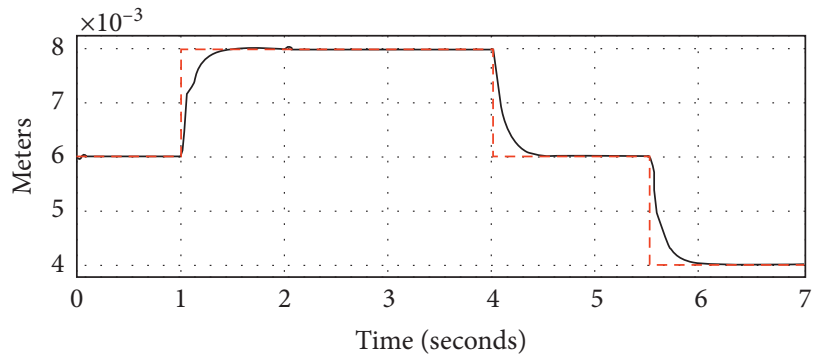

FIgure 5: Continuous: actual ball position $y$. Dashed: desired ball position $y^{*}$.

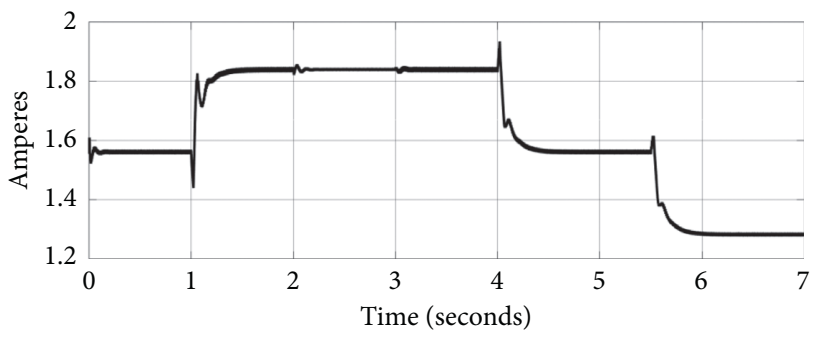

FIGURE 6: Electric current $i$ through the electromagnet.

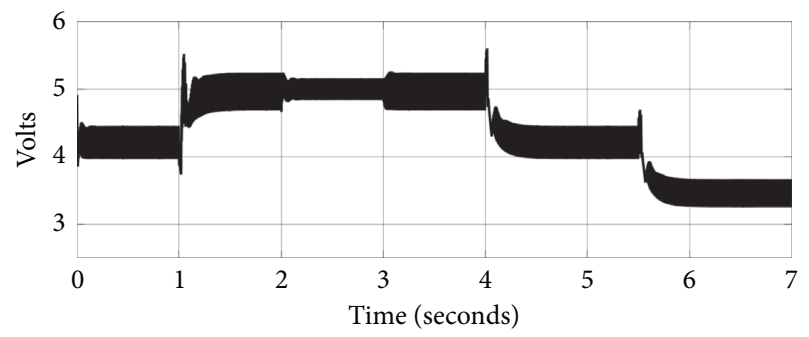

Figure 7: Voltage $v$ at the electromagnet terminals.

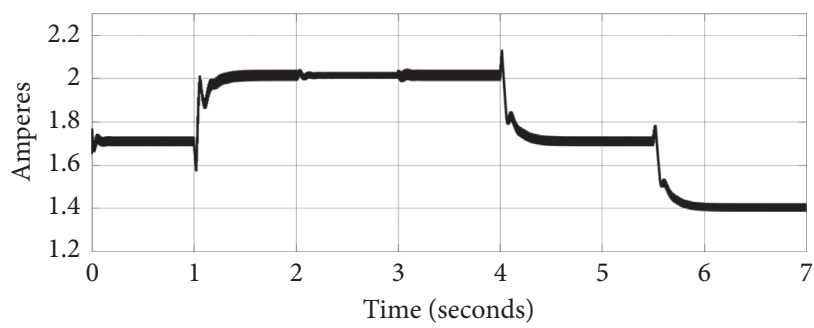

FIgURE 8: Electric current $i_{c}$ through the converter inductor.

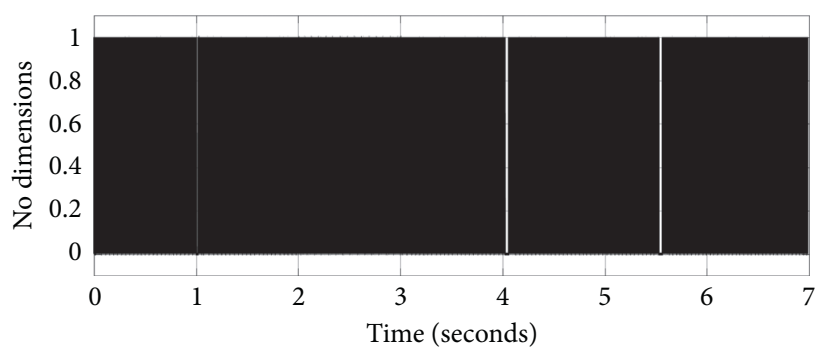

Figure 9: On-off signal $u$ applied at the transistor input. 
freedom in this respect; a larger value for $E$ can be chosen if the system evolution requires more control effort. We have arrived to this conclusion during the simulations that we have performed. For instance, we can observe in Figure 9 three clear zones at $t=1 \mathrm{~s}, t=4 \mathrm{~s}$, and $t=5.5 \mathrm{~s}$ which are evidences that control effort saturates at its extremum values, i.e., either 1 or 0 , when step changes in the desired position are commanded. We also have observed that the ball escapes if larger reference changes are commanded. This is the reason why we have used $E=50 \mathrm{~V}$ for most of the time in the above simulations.

Now, we present some simulation results when using the following classical control scheme:

$$
\begin{aligned}
& v=k_{p i}\left(i^{*}-i\right)+k_{i i} \int_{0}^{t}\left(i^{*}-i\right) \mathrm{d} t, \\
& i^{*}=k_{p}\left(y-y^{*}\right)+k_{d} \dot{y}+k_{i} \int_{0}^{t}\left(y-y^{*}\right) \mathrm{d} t .
\end{aligned}
$$

It is assumed that the DC/DC Buck power electronic converter is not present and a static PWM-based power amplifier is employed. Notice that position error is given as $y-y^{*}$ for the classical PID controller. This is usual in control of magnetic levitation systems. A feature that is required because of the negative gain of the system which, in turn, is produced by the fact that $(\mathrm{d} L(y) / \mathrm{d} y)<0$.

The controller gains were chosen to be $k_{p i}=4.7$, $k_{i i}=100, k_{d}=0.0277, k_{p}=0.866$, and $k_{i}=1.082$. This results in two real dominant closed-loop poles located at $s=-21.5$ and $s=-2.11$. A third real closed-loop pole is at $s=-140$ and two very fast complex conjugate poles, due to the PI control of the electrical dynamics, located at $s=-19 \pm 500 j$.

Our intention is not to compare results obtained with the controller in Proposition 1 and result with the classical control scheme since such a comparison would be unfair. We just want to point out on some features of the classical control scheme. The simulation results are shown in Figures 10-12. The desired position $y^{*}$ is given, again, as in (50).

Notice that the position response has a very short rise time but a large settling time. This is produced by initial fast changes on both the applied voltage and the resulting electric current, which tend slowly to constant values afterwards. Notice that position response exhibits a large overshoot despite the dominant closed-loop poles being real. Recall that the fast complex conjugate poles due to the electrical dynamics cannot produce such a slow overshoot. As it is clearly explained in [31], reason for such a large overshoot is the open-loop unstable pole of the magnetic levitation system which is responsible of its open-loop instability. It is also demonstrated in [31] that this feature cannot be avoided when using the classical control scheme in (51) and (52).

Since classical control schemes rely on linear approximations of the plant to control, it is reasonable to wonder whether some advantages could be obtained when proposing control schemes that take into account more information of the plant nonlinear dynamical model. This is the intention of nonlinear control schemes as the one presented in

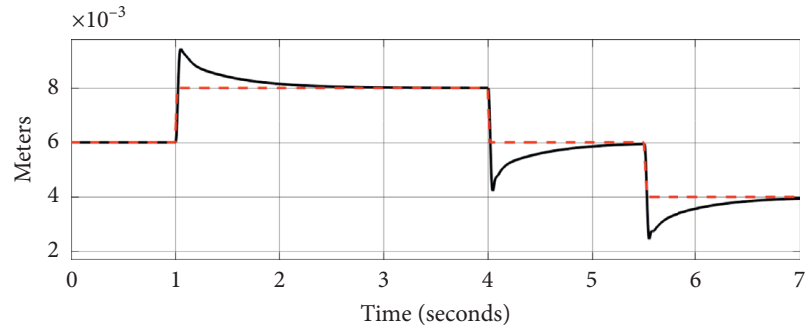

Figure 10: Classical control scheme. Continuous: actual ball position $y$. Dashed: desired ball position $y^{*}$.

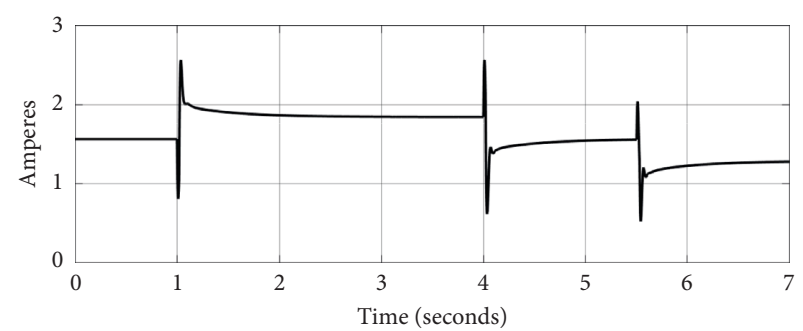

Figure 11: Classical control scheme. Electric current $i$ through the electromagnet.

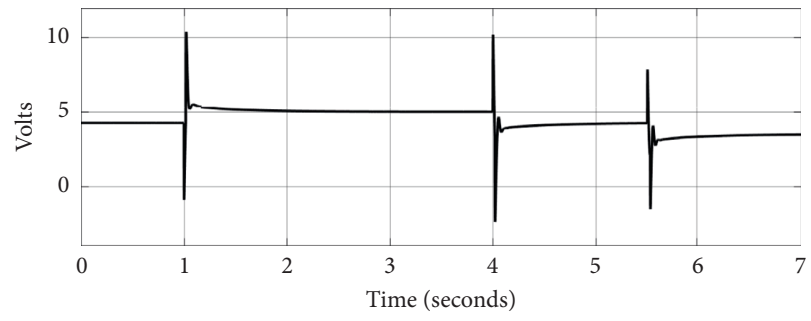

FIgUre 12: Classical control scheme. Voltage $v$ at the electromagnet terminals.

Proposition 1 aside from taking into account some additional dynamics as that of the DC/DC Buck power electronic converter.

Notice that the expressions in (18)-(20) are the nonlinear versions of (51) and (52), i.e., they represent a PI electric current loop and a PID position loop. On the contrary, (16) and (17), represent a sliding mode control loop for electric current at the converter inductor and a nonlinear PI loop for voltage at the converter capacitor. This means that (16) and (17) are additional control loops that are included to cope with system components that are not present in standard magnetic levitation systems. This is what we mean when stating that the control scheme in Proposition 1 is simple if we take into account the complexity of the nonlinear model of the plant.

\section{Conclusions}

In this paper, we have solved the position regulation problem in a magnetic levitation system when it is fed by a DC/DC Buck power electronic converter as power amplifier. 
Contrary to previous works in the literature, which are concerned with control of DC motors, we have solved this problem for the first time for a nonlinear electromechanical system.

This fact represents the merit of our proposal despite the local nature of the results. In this respect, we stress that the region of attraction might be enlarged by including additional nonlinear terms to the control law. However, we have decided not to do this because we are interested in presenting a simple control law in order to render it free of numerical errors and noise amplification.

Our proposal relies on a novel passivity-based approach which exploits the natural energy exchange existing among the mechanical and electrical subsystems that compose the plant. This allows several nonlinear terms to naturally cancel, i.e., without requiring to include additional terms in the control law, which renders simple the control law. Moreover, contrary to previous well known works in the literature, our approach does not rely on proving exponential convergence to zero of the electrical dynamics. This is good news since, otherwise, we would require to online compute and feedback both the time derivative of the desired electric current in the electromagnet and the time derivative of the desired voltage at the electromagnet terminals. Such online computations would render the control law complex and sensitive to numerical errors and noise amplification.

Finally, although the control law is simple, the stability proof may require much attention from the reader. This might be seen as a disadvantage of the approach but it is the authors' belief that it is the role of the control community to cope with mathematical analysis and other related theoretical complications. The important practical thing is that the resulting control scheme is intuitively simple to understand and to implement: a multiloop scheme driven by PI and PID controllers provided with explicit tuning guide lines. We must also say that our approach only considers the regulation task.

\section{Appendix}

\section{A. Conditions for Positive Definiteness of $V_{m}\left(\dot{y}, \tilde{y}, z-m g / k_{i}\right)$, Defined in (40)}

Choose

$$
0<L^{*}<M
$$

and $k_{d}>0$ large enough such that

$$
G(\widetilde{y}) \geq \alpha^{* 2} m H(\widetilde{y}), \quad \forall \widetilde{y} \in \mathscr{R},
$$

where $\alpha^{*}>\alpha>0$ and

$$
\begin{aligned}
& G(\widetilde{y})= \begin{cases}\frac{k_{d}}{2} \widetilde{y}^{2}, & |\widetilde{y}| \leq L^{*}, \\
\frac{k_{d}}{2} L^{* 2}+k_{d} L^{*}\left(\widetilde{y}-L^{*}\right), & \tilde{y}>L^{*}, \\
\frac{k_{d}}{2} L^{* 2}-k_{d} L^{*}\left(\widetilde{y}+L^{*}\right), & \tilde{y}<-L^{*},\end{cases} \\
& H(\tilde{y})= \begin{cases}\tilde{y}^{2}, & |\widetilde{y}| \leq M, \\
M^{2}, & |\widetilde{y}|>M .\end{cases}
\end{aligned}
$$

We stress that (A.2) can always be verified graphically. Define $d=m g / k_{i}, z^{\prime}=z-d$, and

$$
\begin{aligned}
& \Gamma\left(z^{\prime}\right)= \begin{cases}\frac{k_{i}}{2}\left(z^{\prime}\right)^{2}, & -L^{*} \leq z^{\prime}+d \leq L^{*}, \\
\frac{k_{i}}{2}\left(L^{*}-d\right)^{2}+k_{i}\left(z^{\prime}+d-L^{*}\right)\left(L^{*}-d\right), & z^{\prime}+d>L^{*}, \\
\frac{k_{i}}{2}\left(-L^{*}-d\right)^{2}+k_{i}\left(z^{\prime}+d+L^{*}\right)\left(-L^{*}-d\right), & z^{\prime}+d<L^{*},\end{cases} \\
& F\left(z^{\prime}\right)= \begin{cases}z^{\prime 2}, & -M \leq z^{\prime}+d \leq M, \\
(M-d)^{2}, & z^{\prime}+d>M, \\
(-M-d)^{2}, & z^{\prime}+d<-M .\end{cases}
\end{aligned}
$$

Choose a large enough $k_{i}>0$ such that

$$
\Gamma\left(z^{\prime}\right) \geq \alpha^{* 2} \beta^{* 2} m F\left(z^{\prime}\right),
$$

for some $\alpha^{*}>\alpha>0$ and $\beta^{*}>\beta>0$. Notice that (A.5) can always be verified graphically. Function $V_{m}\left(\dot{y}, \tilde{y}, z-m g / k_{i}\right)$ is positive definite and radially unbounded if $k_{p}>0, \alpha>0$, 
and $\beta>0$ and (A.1), (A.2), and (A.5) are satisfied. Proof of this result is presented in [30].

\section{B. Some Algebraic Relations for Proof of Proposition 1}

Notice the following:

$$
\left|i^{*}\right| \leq \sqrt{\frac{2}{|\mathrm{~d} L(y) / \mathrm{d} y|_{\min }}\left(k_{p} M+k_{d}|\dot{y}|+k_{i} M\right)}=I^{*}(|\dot{y}|),
$$

where (19) has been employed. On the other hand, according to the Mean Value Theorem:

$$
0 \leq I^{*}(|\dot{y}|)-I^{*}(0)=\left.\frac{\mathrm{d} I^{*}(|\dot{y}|)}{\mathrm{d}|\dot{y}|}\right|_{|\dot{y}|=\zeta}(|\dot{y}|-0),
$$

$$
\left.\frac{\mathrm{d} I^{*}(|\dot{y}|)}{\mathrm{d}|\dot{y}|}\right|_{|\dot{y}|=\zeta}>0, \quad \forall \zeta>0
$$

i.e.,

$$
I^{*}(|\dot{y}|)=\left.\frac{d I^{*}(|\dot{y}|)}{d|\dot{y}|}\right|_{|\dot{y}| t=n \zeta}|\dot{y}|+I^{*}(0),
$$

for some $\zeta>0$ belonging to the line joining the points $|\dot{y}|$ and 0. Hence, from (B.1) and (B.3), we find

$$
\begin{aligned}
\left|i^{*}\right| & \leq k_{\delta}|\dot{y}|+I^{*}(0), \\
k_{\delta} & =\max _{|\dot{y}|>0}\left\{\frac{\mathrm{d} I^{*}(|\dot{y}|)}{\mathrm{d}|\dot{y}|}\right\} .
\end{aligned}
$$

Notice that $k_{\delta}$ and $I^{*}(0)$ are positive and finite. This allows to write

$$
\begin{aligned}
\left|\frac{\mathrm{d} L(y) \tilde{i}^{2} i^{*}}{\mathrm{~d} y}\right| & \leq k_{\sigma} \widetilde{i}^{2}\left(k_{\delta}|\dot{y}|+I^{*}(0)\right), \\
\left|\alpha \frac{\mathrm{d} L(y)}{\mathrm{d} y} h(\widetilde{y}) \widetilde{i}^{*}\right| & \leq \alpha k_{\sigma} k_{\delta} M|\widetilde{i}||\dot{y}|+\alpha k_{\sigma} \widetilde{i}|| h(\widetilde{y})\left|I^{*}\right| 0 \mid, \\
\left|\alpha \beta \frac{\mathrm{d} L(y)}{\mathrm{d} y} s(z) \widetilde{i i}^{*}\right| & \leq 2 \alpha \beta M k_{\sigma} k_{\delta}|\widetilde{i}||\dot{y}|+\alpha \beta k_{\sigma} \widetilde{i}|| s(z) \mid I^{*}(0), \\
k_{\sigma} & =\max \left\{\left|\frac{\mathrm{d} L(y)}{\mathrm{d} y}\right|\right\} .
\end{aligned}
$$

We stress that $k_{\sigma}>0$ is always finite, i.e., the maximal value of $|\mathrm{d} L(y) / \mathrm{d} y|$ appears when $y=0$. On the contrary, we have that at the equilibrium point $i^{*}=i_{e}^{*}$, where

$$
i_{e}^{*}=\sqrt{\frac{2 m g}{\left|\mathrm{~d} L\left(y^{*}\right) / \mathrm{d} y\right|}}
$$

Hence, if we assume that

$$
\begin{aligned}
i^{*} & =i^{*}(h(\tilde{y}), \dot{y}, s(z)) \\
& =\sqrt{\frac{2}{|\mathrm{~d} L(y) / \mathrm{d} y|}\left[k_{p} h(\tilde{y})+k_{d} \dot{y}+k_{i} s(z)+m g\right]},
\end{aligned}
$$

then $i_{e}^{*}=i^{*}(0,0,0)$. Thus, according to the Mean Value Theorem

$$
\begin{aligned}
i^{*}-\sqrt{\frac{2 m g}{\left|\mathrm{~d} L\left(y^{*}\right) / \mathrm{d} y\right|}} & =i^{*}(h(\tilde{y}), \dot{y}, s(z))-i^{*}(0,0,0) \\
& =\left.\frac{\partial i^{*}(x)}{\partial x}\right|_{x=p}\left((h(\tilde{y}), \dot{y}, s(z))^{T}-(0,0,0)^{T}\right),
\end{aligned}
$$

for some $p$ belonging to the line joining the points $(h(\tilde{y}), \dot{y}, s(z))$ and $(0,0,0)$. Finally, recalling that $\left|v^{T} w\right| \leq\|v\|\|w\|$, for all $v, w \in \mathscr{R}^{n}$ and $\|x\| \leq\|x\|_{1}$, we can write

$$
\left|i^{*}-\sqrt{\frac{2 m g}{\left|\mathrm{~d} L\left(y^{*}\right) / \mathrm{d} y\right|}}\right| \leq\left(k^{*}+k_{t}|\dot{y}|\right)[|h(\tilde{y})|+|\dot{y}|+|s(z)|],
$$

where the constants $k^{*}$ and $k_{t}$ are defined from the norm of the following vector:

$$
\begin{aligned}
\frac{\partial i^{*}(x)}{\partial x}= & \left(\frac{2 F^{*}}{|\mathrm{~d} L(y) / d y|}\right)^{-1 / 2} \times\left\{[1,0,0]\left(\frac{\mathrm{d}}{\mathrm{d} y}\left|\frac{\mathrm{d} L(y)}{\mathrm{d} y}\right|^{-1}\right)\right. \\
& \times \frac{\mathrm{d}\left(y^{*}+\tilde{y}\right)}{\mathrm{d} \widetilde{y}} \frac{\mathrm{d} \tilde{y}}{\mathrm{~d} h(\tilde{y})}\left[k_{p} h(\widetilde{y})+k_{i} s(z)+m g\right] \\
& \left.+\left|\frac{\mathrm{d} L(y)}{\mathrm{d} y}\right|^{-1}\left[k_{p}, k_{d}, k_{i}\right]\right\} \\
& +\left(\frac{2 F^{*}}{|\mathrm{~d} L(y) / \mathrm{d} y|}\right)^{-1 / 2}[1,0,0]\left(\frac{\mathrm{d}}{\mathrm{d} y}\left|\frac{\mathrm{d} L(y)}{\mathrm{d} y}\right|^{-1}\right) \\
& \times \frac{\mathrm{d}\left(y^{*}+\tilde{y}\right)}{\mathrm{d} \widetilde{y}} \frac{\mathrm{d} \tilde{y}}{\mathrm{~d} h(\tilde{y})} k_{d} \dot{y},
\end{aligned}
$$

recalling that, according to $(21), \mathrm{d} \widetilde{y} / \mathrm{d} h(\tilde{y})$ is bounded, $|\mathrm{d} L(y) / \mathrm{d} y|>0, \quad\left(2 F^{*} /(|\mathrm{d} L(y) / \mathrm{d} y|)\right)^{-1 / 2}$, and $\mathrm{d} / \mathrm{d} y \mid \mathrm{d} L(y) /$ $\left.\mathrm{d} y\right|^{-1}$ are bounded, and $|h(\widetilde{y})| \leq M$ and $|s(z)| \leq 2 M$. Thus, $k^{*}$ and $k_{t}$ are finite.

\section{Data Availability}

The data used to support the findings of this study are available from the corresponding author upon request.

\section{Conflicts of Interest}

The authors declare that there are no conflicts of interest regarding the publication of this article. 


\section{Acknowledgments}

The processing charge of this article was generously supported by the Instituto Politécnico Nacional, México. The work of V. M. Hernández-Guzmán was supported by the SNI-México. The work of R. Silva-Ortigoza and M. Marciano-Melchor was supported by the SNI-México and IPN programs EDI and SIBE.

\section{References}

[1] F. Antritter, P. Maurer, and J. Reger, "Flatness based control of a buck-converter driven DC motor," in Proceedings of the 4th IFAC Symposium on Mechatronic Systems, Heidelberg, Germany, September 2006.

[2] S. E. Lyshevski, Electromechanical Systems, Electric Machines, and Applied Mechatronics, CRC Press, Boca Raton, FL, USA, 1999.

[3] I. Boldea and S. A. Nasar, Electric Drives, CRC Press LLC, Boca Raton, FL, USA, 1999.

[4] H. El Fadil and F. Giri, "Accounting of DC-DC power converter dynamics in DC motor velocity adaptive control," in Proceedings of the IEEE International Conference on Control Applications, pp. 3157-3162, Munich, Germany, October 2006.

[5] J. Linares-Flores and H. Sira-Ramírez, "A smooth starter for a DC machine: a flatness based approach," in Proceedings of the 1st International Conference on Electrical and Electronics Engineering, pp. 589-594, Acapulco, Mexico, September 2004.

[6] J. Linares-Flores and H. Sira-Ramírez, "Sliding mode-delta modulation GPI control of a DC motor through a buck converter," in Proceedings of the 2nd IFAC Symposium on System, pp. 405-409, Oaxaca, Mexico, December 2004.

[7] J. Linares-Flores and H. Sira-Ramírez, "DC motor velocity control through a DC-to-DC power converter," in Proceedings of the 43rd IEEE Conference on Decision and Control, vol. 5, pp. 5297-5302, Atlantis, The Bahamas, December 2004.

[8] R. Silva-Ortigoza, J. R. García-Sánchez, J. M. Alba-Martínez et al., "Two-stage control design of a buck converter/DC motor system without velocity measurements via $a \Sigma-\Delta$ modulator," Mathematical Problems in Engineering, vol. 2013, Article ID 929316, 11 pages, 2013.

[9] R. Silva-Ortigoza, C. Márquez-Sánchez, F. Carrizosa-Corral, M. Antonio-Cruz, J. M. Alba-Martínez, and G. SaldañaGonzález, "Hierarchical velocity control based on differential flatness for a DC/DC Buck converter-DC motor system," Mathematical Problems in Engineering, vol. 2014, Article ID 912815, 12 pages, 2014.

[10] R. Silva-Ortigoza, V. M. Hernandez-Guzman, M. AntonioCruz, and D. Munoz-Carrillo, "DC/DC Buck power converter as a smooth starter for a DC motor based on a hierarchical control," IEEE Transactions on Power Electronics, vol. 30, no. 2, pp. 1076-1084, 2015.

[11] H. Sira-Ramirez and M. A. Oliver-Salazar, "On the robust control of buck-converter DC-motor combinations," IEEE Transactions on Power Electronics, vol. 28, no. 8, pp. 39123922, 2013.

[12] R. Sureshkumar and S. Ganeshkumar, "Comparative study of proportional integral and backstepping controller for buck converter," in Proceedings of the International Conference on Emerging Trends in Electrical and Computer Technology, pp. 375-379, Tamil Nadu, India, March 2011.

[13] E. Hernandez-Marquez, R. Silva-Ortigoza, J. R. Garcia-Sanchez, M. Marcelino-Aranda, and G. Saldana-Gonzalez, "A
DC/DC buck-boost converter-inverter-DC motor system: sensorless passivity-based control," IEEE Access, vol. 6, pp. 31486-31492, 2018.

[14] E. Hernández-Márquez, J. R. García-Sánchez, R. Silva-Ortigoza et al., "Bidirectional tracking robust controls for a DC/ DC buck converter-DC motor system," Complexity, vol. 2018, Article ID 1260743, 10 pages, 2018.

[15] R. Silva Ortigoza, J. N. Alba Juarez, J. R. Garcia Sanchez, M. Antonio Cruz, V. M. Hernandez Guzman, and H. Taud, "Modeling and experimental validation of a bidirectional DC/ DC buck power electronic converter-DC motor system," IEEE Latin America Transactions, vol. 15, no. 6, pp. 1043-1051, 2017.

[16] V. M. Hernández-Guzmán, R. Silva-Ortigoza, and D. MuñozCarrillo, "Velocity control of a brushed DC-motor driven by a DC to DC buck power converter," International Journal of Innovative Computing, Information and Control, vol. 11, no. 2, pp. 509-521, 2015.

[17] Z. Chen, W. Gao, and X. Ye, "Frequency domain closed-loop analysis and sliding mode control of a nonminimum phase buck-boost converter," Control and Intelligent Systems, vol. 38, no. 4, pp. 245-255, 2010.

[18] Z. Chen, J. Hu, and W. Gao, "Closed-loop analysis and control of a non-inverting buck-boost converter," International Journal of Control, vol. 83, no. 11, pp. 2294-2307, 2010.

[19] Z. Chen, W. Gao, J. Hu, and X. Ye, "Closed-loop analysis and cascade control of a nonminimum phase boost converter," IEEE Transactions on Power Electronics, vol. 26, no. 4, pp. 1237-1252, 2011.

[20] R. Ortega, A. Loría, P. Nicklasson, and H. Sira-Ramírez, Passivity-based Control of Euler-Lagrange Systems, Springer, London, UK, 1998.

[21] R. Ortega, A. J. van der Schaft, I. Mareels, and B. Maschke, "Putting energy back in control," IEEE Control Systems Magazine, vol. 21, no. 2, pp. 18-33, 2001.

[22] H. Rodriguez, R. Ortega, and I. Mareels, "Nonlinear control of magnetic levitation systems via energy balancing," in Proceedings of the American Control Conference, Chicago, IL, USA, June 2000.

[23] V. M. Hernández-Guzmán and R. Silva-Ortigoza, Automatic Control with Experiments, Springer, Cham, Switzerland, 2019.

[24] A. Astolfi and R. Ortega, "Immersion and invariance: a new tool for stabilization and adaptive control of nonlinear systems," IEEE Transactions on Automatic Control, vol. 48, no. 4, pp. 590-606, 2003.

[25] A. Astolfi, D. Karagiannis, and R. Ortega, Nonlinear and Adaptive Control with Applications, Springer, Cham, Switzerland, 2008.

[26] R. S. Ortigoza, J. N. A. Juarez, J. R. G. Sanchez, V. M. H. Guzman, C. Y. S. Cervantes, and H. Taud, "A sensorless passivity-based control for the DC/DC buck converter-inverter-DC motor system," IEEE Latin America Transactions, vol. 14, no. 10, pp. 4227-4234, 2016.

[27] V. M. Hernández-Guzmán and R. Silva, "Current loops in a magnetic levitation system," International Journal of Innovative Computing, Information and Control, vol. 5, no. 5, pp. 1275-1283, 2009.

[28] A. Zavala-Rio and V. Santibanez, "A natural saturating extension of the PD-with-desired-gravity-compensation control law for robot manipulators with bounded inputs," IEEE Transactions on Robotics, vol. 23, no. 2, pp. 386-391, 2007.

[29] H. K. Khalil, Nonlinear Systems, Prentice-Hall, Upper Saddle River, NJ, USA, 3rd edition, 2002. 
[30] V. M. Hernández-Guzmán and J. Orrante-Sakanassi, "Global PID control of robot manipulators equipped with PMSMs," Asian Journal of Control, vol. 20, no. 1, pp. 236-249, 2018.

[31] G. C. Goodwin, S. F. Graebe, and M. E. Salgado, Control System Design, Prentice-Hall, Upper-Saddle River, NJ, USA, 2001. 